FORMATION Formation emploi

Revue française de sciences sociales

99 | juillet-septembre 2007

Les usages sociaux de la compétence

\title{
De la formation professionnelle à la professionnalité d'une formation
}

From vocational training to its professionalization

Von der beruflichen Bildung zur Professionalität der Berufsbildung

Catherine Béduwé, Jean-Michel Espinasse et Jean Vincens

\section{OpenEdition}

\section{Journals}

Édition électronique

URL : http://journals.openedition.org/formationemploi/1495

DOI : 10.4000/formationemploi.1495

ISSN : 2107-0946

Éditeur

La Documentation française

Édition imprimée

Date de publication : 1 juillet 2007

Pagination : 103-120

ISSN : 0759-6340

Référence électronique

Catherine Béduwé, Jean-Michel Espinasse et Jean Vincens, « De la formation professionnelle à la professionnalité d'une formation », Formation emploi [En ligne], 99 | juillet-septembre 2007, mis en ligne le 30 septembre 2009, consulté le 30 octobre 2020. URL : http://journals.openedition.org/

formationemploi/1495; DOI : https://doi.org/10.4000/formationemploi.1495

(c) Tous droits réservés 


\title{
Formation
}

\section{De la formation professionnelle à la professionnalité d'une formation}

Par Catherine Béduwé, Jean-Michel Espinasse, Jean Vincens*

\begin{abstract}
Comment expliquer que les formations dites professionnelles ne débouchent pas plus souvent sur les emplois prévus? Comment expliquer que les jeunes formés

à ces emplois ne gagnent pas plus que les autres?

Cet article propose une mesure des liens formations-emplois, différente de l'approche classique basée sur l'adéquation.
\end{abstract}

Le développement de la formation professionnelle et la professionnalisation des formations à tous les niveaux d'enseignement, y compris à l'université, apparaissent comme des exigences fortes de notre société. Mieux former les jeunes aux besoins de l'économie faciliterait leur insertion professionnelle et résoudrait une grande partie des problèmes de chômage. Il s'agit donc de diversifier l'offre de formation et de promouvoir l'allongement des études de manière à doter les nouvelles générations des compétences professionnelles et des capacités d'adaptation nécessaires aux entreprises pour faire face aux évolutions technologiques de plus en plus rapides. Il ne s'agit pas seulement de former plus longtemps les jeunes mais bien de leur donner, à tous les niveaux d'études, des compétences spécifiques indispensables à l'exercice d'une activité professionnelle bien identifiée.

Cette évolution semble validée par une série d'analyses quantitatives qui montrent que les diplômés d'une formation professionnelle (i.e. filière professionnelle telle que DUT, BTS, IUP, MST, DESS ${ }^{1} \ldots$ )

${ }^{1}$ Diplôme universitaire de technologie (DUT), brevet de technicien supérieur (BTS), Institut universitaire professionnalisé (IUP), maîtrise de sciences et techniques (MST), diplôme d'études supérieures spécialisées (DESS). ont une insertion plus performante que les jeunes qui se présentent sur le marché du travail avec une formation générale (i.e. les autres filières): accès plus rapide au premier emploi, moindre chômage dans les premières années de vie active, stabilisation plus grande et plus rapide des emplois, meilleure rémunération ( $c f$. par exemple Giret et Moullet, 2005).

Catherine Béduwé est ingénieur de recherches au LIRHE (Laboratoire interdisciplinaire de recherches sur les ressources humaines et l'emploi) Ses travaux portent sur l'insertion professionnelle des jeunes et la relation formation-emploi. Elle a récemment coordonné un rapport pour le ministère de l'Éducation nationale sur les filières scientifiques et l'emploi.

Jean-Michel Espinasse est économiste au LIRHE. II travaille sur la relation formation-emploi et sur les problèmes de certification professionnelle.

Jean Vincens est professeur émérite à l'université des sciences sociales de Toulouse. II a publié récemment : "L'adéquation formation-emploi » in J.-F. Giret, A. Lopez, J. Rose, Des formations pour quels emplois? La Découverte, Cereq, 2005 pp. 149-162 ; "Un diplôme et un emploi pour chaque étudiant? », Note Lirhe 437, septembre 2006. 
L'argument est il suffisant? La plupart des formations professionnelles, notamment supérieures, sélectionnent leurs étudiants à l'entrée, tout en permettant la poursuite d'études vers des formations générales. Ces formations attirent de très bons élèves, souvent très motivés, à qui elles offrent la possibilité de construire des parcours de formation complexes et performants en matière d'insertion professionnelle (DUT puis master spécialisé par exemple, ou école d'ingénieur puis doctorat). Les diplômes généraux sont à la fois le couronnement d'études ouvrant sur les emplois de l'enseignement et de la recherche ou donnant accès aux concours de la fonction publique (ce qui en fait des formations professionnelles au même titre que les autres) et le signe d'études « inachevées » ou abandonnées faute d'orientation vers des filières professionnelles. Par ailleurs, la complexité croissante des parcours individuels de formation affaiblit considérablement le clivage entre formations professionnelles et générales : maîtrise générale suivie d'un DESS par exemple, ou encore instauration de stages professionnels en formation générale... (Giret, Moullet, Thomas, 2003). La comparaison tranchée entre diplômes professionnels et diplômes généraux ne va donc pas sans poser problème.

Mais l'essentiel n'est pas là. On considère généralement que les formations professionnelles sont construites en référence à un métier, une profession, et qu'elles délivrent les compétences nécessaires à l'exécution de ce métier ou cette profession (définition proposée par la Cour de Justice des Communautés européennes, 1985) supposés clairement définis et bien identifiés (Vincens, Chirache, 1996). Implicitement, les bonnes performances des formations professionnelles sont alors attribuées aux efforts de rapprochement entre système de formation et système productif. Cette construction en partenariat des formations devrait conduire à produire les qualifications/ compétences nécessaires aux besoins de l'économie, en conformité avec la demande des employeurs. L'évaluation de ces formations doit donc aller au-delà de la notion de filière (IUT, DESS, etc.) et tenir compte de la spécialité professionnelle de chaque formation ou diplôme. Un avocat n'est pas un ingénieur agronome, un plombier n'est pas un coiffeur, mais tous sortent d'une formation professionnelle... L'analyse des performances de ces formations doit traiter de la correspondance entre ces formations (définies en termes de niveau et de spécialité) et les emplois auxquels elles mènent (définis en termes de catégorie sociale et de domaine professionnel). L'hypothèse sous-jacente à la professionnalisation est que la correspondance formations-emplois étant meilleure pour ces formations bien ciblées professionnellement, leurs performances doivent être meilleures. Pourtant, comme on va le montrer, nombre d'analyses mettent en évidence la faible correspondance entre spécialité de formation et spécialité d'emploi. Comment expliquer ce résultat?

Cet article tente de répondre à cette question en confrontant deux manières de formaliser la correspondance entre formations et emplois et les évaluations qui en découlent

Dans une première section, nous présentons une approche normative (ou a priori) des relations entre formations et emplois et montrons ses liens avec la notion de métier. L'évaluation de la correspondance teste l'adéquation entre formations et emplois. Dans la deuxième, nous présentons une approche statistique (ou a posteriori) de ces relations articulée autour de la notion de compétences. Nous utiliserons des indices de concentration (indices de Gini) pour caractériser la professionnalité (le degré de professionnalisation) d'une formation. La troisième section illustre brièvement comment les deux approches renvoient à une lecture différente des observations d'insertion professionnelle dans l'enquête Génération 98. La dernière compare les deux approches dans le cadre d'une équation de salaire. Les deux méthodes ne s'excluent pas. La seconde s'inscrit dans une perspective plus générale d'appréhension des liens entre formations et emplois et permet, notamment, de passer de la notion dichotomique de formation professionnelle à celle, beaucoup plus continue, de professionnalité d'une formation.

\section{DU MÉTIER À UNE APPROCHE NORMATIVE DE LA RELATION FORMATIONS-EMPLOIS}

L'ouvrage Quelles formations pour quels emplois que le Céreq vient de publier (Giret, Lopez et Rose, 
2005), contredit l'hypothèse d'une forte correspondance emplois-formations à l'issue d'une formation professionnelle :

À partir du niveau CAP (certificat d'aptitude professionnelle), moins d'un jeune sur deux exerce un emploi dont la spécialité est en accord formel avec celle de sa formation (Couppié, Giret, Lopez, 2005, p. 94). Les entreprises recrutent donc des jeunes dont la spécialité de formation ne correspond pas à celle de l'emploi offert. Ce faible taux s'observe en conjoncture défavorable (92-97) mais également favorable (98-2003) ; il ne s'améliore que très légèrement au fil des cinq premières années de vie professionnelle. Cette faible correspondance entre métier appris et métier exercé a été mise en évidence pour l'ensemble des actifs par Dumartin (1997) et a incité les analystes à ne conduire des études que sur des segments bien délimités du système de formation et/ ou d'emploi, là où la question de l'adéquation faisait sens. Ce type de résultat a également conduit à douter de la pertinence des tentatives de planification de l'éducation, même à des niveaux de spécialité très regroupés des formations et des emplois.

Cette faible correspondance existe également si on prend en compte les seules formations professionnelles. Ainsi, selon les filières, entre $45 \%$ et $61 \%$ des diplômés de l'enseignement supérieur professionnel accèdent à un emploi dont le domaine professionnel correspond à leur spécialité de formation (Giret et $a l, 2003$ ). Ces taux sont - évidemment supérieurs à ceux des diplômés des filières générales (ente $15 \%$ et $46 \%$ ) mais les écarts apparaissent moins importants qu'attendu (Giret et Moullet 2005, $\mathrm{p}$ 121). En outre, le caractère professionnel d'une formation ne semble pas constituer une protection déterminante contre le déclassement (p. 124), ce qui, d'une certaine manière, réfute le pouvoir de consensus conféré à la formation professionnelle : «les attentes des étudiants et celles des utilisateurs des futurs diplomés sont convergentes en ce qui concerne le niveau de recrutement $»$ (Vincens et Chirache, 1996).

En outre, exercer le métier appris est globalement peu rentable $(+1.6 \%$ de salaire en moyenne et toutes choses égales par ailleurs pour les «adéquats ») et seulement très rentable pour très peu d'individus (ceux qui sont par ailleurs surclassés à des niveaux élevés) (Bruyère et Lemistre, 2005). Ainsi, selon ces résultats, il n'y aurait pas de véritable avantage salarial à exercer le métier que l'on a appris (il y en a peut-être d'autres). Ce qui voudrait dire aussi que le coût d'adaptation d'un salarié lié à l'acquisition des compétences techniques nécessaires à l'exercice d'un emploi pour lequel il n'est pas formé serait globalement - assez faible.

Les deux premiers résultats (faible correspondance entre spécialité de formation et spécialité d'emploi) ne sont contre-intuitifs que pour tous ceux qui ont une vision très normative ou adéquationniste des relations entre formations et emplois. Les autres connaissent cette réalité récurrente et savent bien qu'il ne s'agit en rien d'un artefact statistique. Il ne s'agit pas non plus d'un disfonctionnement du marché du travail et/ou du système de formation: l'accès à l'emploi est un processus complexe où la formation explicite (le diplôme) est une condition souvent nécessaire mais rarement suffisante. Il est cependant clair qu'il faut prendre acte de ce que «... le lien entre les formations (professionnelles) et les types d'emplois sur lesquels elles débouchent n'est pas statistiquement étroit » (Giret et al., 2003) et mettre ce résultat au centre de la réflexion.

Le troisième résultat (faible avantage salarial aux adéquats) pose plus de questions. Il oblige à s'interroger sur les fondements mêmes de la professionnalisation : on peut comprendre pourquoi un individu n'occupe pas l'emploi pour lequel il a été formé. On comprend moins bien pourquoi, quand il l'obtient, il n'en tire que fort peu d'avantages pécuniaires. On comprend encore moins comment des individus non (directement) formés à des emplois qualifiés peuvent obtenir une rémunération équivalente à celles des individus (directement) formés. Si posséder formellement les compétences nécessaires à l'exercice d'un métier ne confère pas d'avantage décisif en termes de salaire, à quoi bon diversifier les formations ?

Ces résultats sont le produit d'une démarche qui repose, implicitement, sur une représentation normative des relations entre formations et emplois, entre spécialité de formation et spécialité d'emploi. Même si les auteurs mettent en garde contre l'utilisation de ce type de modèle pour analyser l'accès à l'emploi 
des jeunes et le marché du travail des débutants, l'évaluation des formations peine à s'en écarter. Dans sa critique de l'ouvrage (auquel nous avons largement contribué...) C. Frétigné évoque l'idée d'un «adéquationnisme méthodologique », ce qui lui fait écrire que "Le "paradigme" adéquationniste reste bien le cadre princeps des analyses, une sorte de ligne d'horizon infranchissable et indépassable» (Frétigné, 2006).

La tentation est d'autant plus grande d'utiliser ce type de modèle dans l'analyse des formations professionnelles et donc des relations entre spécialités de formation et d'emploi qu'il est construit autour de la notion de métier, celle-là même qui fonde la formation professionnelle: tout emploi qualifié est un "métier» qui doit avoir été appris (voire même appris à l'école dans le cas des débutants) pour pouvoir être exercé. Le jeune qui n'exerce pas le métier qu'il a appris a toutes les chances de connaître une insertion professionnelle plus longue, plus difficile et d'occuper un emploi non qualifié. L'entreprise qui ne trouve pas sur le marché l'individu déjà formé supporte un coût élevé de mise à niveau et donc une baisse d'efficacité. Une partition de l'ensemble des emplois en métiers induit logiquement une partition des individus en fonction du métier appris. L'analyse des relations formations-emplois se focalise alors sur la mise en correspondance entre métiers exercés et métiers appris. Les situations d'adéquation optimisent les avantages pour les individus, les employeurs et la collectivité qui a financé la formation. Les situations de non-correspondance pointent le disfonctionnement dans l'allocation des ressources humaines.

Pourtant, des travaux comme ceux de Kirsch et Werquin (1995) ont montré que cette dichotomie correspondance/non-correspondance rendait très mal compte de la diversité des situations observées. Grâce à l'analyse des trajectoires professionnelles, Bruyère, Espinasse \& Fourcade (2004) mettent en évidence l'existence de trois groupes de parcours à l'issue d'une formation: les "adéquats ", les situations de grande précarité professionnelle dominées par le chômage et/ou l'emploi non qualifié, et des «non-adéquats » caractérisés par des parcours hors du champ professionnel correspondant aux savoirs acquis en formation. Le niveau de formation, le taux d'emplois qualifiés, les salaires de ces jeunes ne sont pas fondamentalement différents de ceux des " adéquats ». Le problème est donc celui de l'hétérogénéité des cas de non-correspondance: «La non correspondance cesse effectivement d'être un problème dès lors que la correspondance n'incarne plus la normalité mais qu'elle constitue un état parmi d'autres possibles, en fonction des conditions économiques prévalentes » (Stanckewicz, 2006).

\section{DE LA COMPÉTENCE À UNE APPROCHE STATISTIQUE DE LA RELATION FORMATIONS-EMPLOIS}

Dépasser l'approche adéquationniste impose de repartir de l'observation générale, c'est-à-dire de la diversité des relations entre formations et emplois. Les individus ont des savoirs et des compétences différents, y compris à l'issue de la même formation, qui évoluent dans le temps. Les emplois sont des ensembles de tâches imparfaitement descriptibles, qui évoluent avec le temps et selon les caractéristiques des individus qui les occupent. La qualité de l'appariement entre un individu et un emploi dépend de la complétude entre savoirs acquis et savoirs requis. Cette qualité n'est pas postulée a priori mais résulte de l'observation des appariements individuels entre la formation détenue et l'emploi occupé. Il faut pour cela s'appuyer sur des données d'enquêtes et trouver des indicateurs statistiques de cette qualité.

\section{Les compétences pour expliquer la diversité des relations formations-emplois}

La littérature sur la (ou les) compétence(s) conduit à considérer la notion de métier avec prudence. Le travail humain et l'organisation de la firme sont postulés complexes et singuliers. L'individu est décrit comme une collection de savoirs et de savoirfaire plus ou moins indépendants entre eux. L'emploi est également décrit comme nécessitant une collection de savoirs et de savoir-faire articulés autour des 
pratiques et des routines de la firme. Le couplage entre ces deux collections est toujours singulier. Son efficacité productive dépend de la façon dont les deux collections (compétences requises et compétences disponibles) sont mobilisées.

Le travail est envisagé comme une interaction entre le travailleur et son environnement (ses collègues, le capital technique, l'organisation, la culture, le marché...). Les compétences des individus se valorisent uniquement dans une acculturation à l'environnement technico-organisationnel. Cette valorisation est toujours endogène à la firme et elle a un coût que l'entreprise cherche à minimiser sans pouvoir s'en affranchir totalement.

Les acquis de la formation initiale sont au cœur de la compétence d'un débutant, même s'ils n'en constituent qu'une fraction. La formation initiale est donc l'élément déterminant du choix d'un débutant. Chaque jeune possède, de par sa formation, des compétences qu'il a en commun avec d'autres jeunes issus d'autres formations, et des compétences spécifiques que sa formation est seule à délivrer. Elles sont acquises progressivement au fil des cursus. Cela est vrai pour les formations professionnelles mais aussi pour l'ensemble des formations au-delà du tronc commun. Ces compétences communes à plusieurs formations sont extrêmement diverses. Elles découlent de savoirs proprement dits mais également des capacités cognitives acquises.

L'existence de savoirs ou compétences communs à plusieurs formations crée des zones de concurrence entre diplômés de ces formations pour l'accès à certains emplois. Posséder des compétences communes les rend éligibles aux emplois qui mobilisent ces compétences et qui ne font pas prévaloir leurs compétences spécifiques. Ces zones de concurrence sont aussi nombreuses que les formations et les compétences acquises sont diverses. Les acquis spécifiques à chaque formation créent au contraire une zone d'avantage concurrentiel propre à la formation. Recevoir une formation, c'est à la fois être mis en concurrence avec les élèves d'une ou plusieurs autres formations et se protéger de la concurrence que ceux-ci exercent. C'est être préparé prioritairement à certains emplois mais aussi acquérir la possibilité d'accéder à d'autres emplois, quitte à subir la concurrence des jeunes possédant des compétences communes. C'est enfin et surtout acquérir des savoir-être et des réflexes cognitifs pouvant être réutilisés dans un très grand nombre de contextes.

Un recrutement se fait à deux et chacun doit y trouver son intérêt. C'est la règle du double avantage comparatif (Vincens, 2005) : un employeur embauche pour un emploi (qualifié) un individu qui va pouvoir, rapidement et à moindre coût, « produire » les savoir-faire concrets nécessaires. Pour cela, l'employeur considère l'ensemble des compétences de l'individu et non pas seulement les connaissances spécifiques qu'il a acquises dans sa formation. Ces dernières sont parfois déterminantes mais il n'y a aucune raison que ce soit toujours le cas. De son côté, le salarié cherche parmi tous les emplois qui lui sont accessibles ceux qui valorisent le mieux ses compétences. Il se peut que ces emplois ne fassent pas partie de la cible à laquelle ses formateurs avaient pensé. Ce recrutement est un compromis qui se fait aux conditions de marché, compte tenu de l'état des prix et des quantités. Il est donc daté.

D'autres compétences, périphériques aux savoir-faire techniques de l'individu, observées ou non, peuvent expliquer le recrutement. Ainsi, un diplômé d'études supérieures en statistiques peut se trouver en concurrence avec un diplômé d'études supérieures en sciences économiques pour un emploi de chargé d'études financières parce que l'un comme l'autre détiennent les compétences clés pour s'adapter dans des conditions correctes à cet emploi (maîtrise de la modélisation et de son support informatique par exemple). Aucun des deux ne possède pas - pour l'instant - l'ensemble des savoirs concrets produits par cette activité précise (par exemple les produits financiers de la firme) mais ils sont jugés capables de les obtenir rapidement. Chacun détient des compétences spécifiques qui peuvent - éventuellement constituer, aux yeux de l'employeur, des avantages décisifs. Si ce n'est pas le cas, le choix se fera sur d'autres critères (comportement, réseaux, savoirêtre...) qui ne relèvent pas - directement - de la formation et qui ne peuvent être observés. Un seul des deux sera recruté et laissera une trace dans le système d'observation statistique. 


\section{Une approche statistique pour modéliser les relations formations-emplois}

La notion de compétences invite à considérer les travailleurs comme partiellement substituables pour un emploi, et réciproquement. Elle s'oppose à une approche métier qui les envisage comme des produits non substituables. Elle présente l'avantage de prendre acte de la dispersion observée dans les relations formations-emplois sans la postuler comme le résultat d'un déséquilibre ou d'un dysfonctionnement. Elle considère toujours qu'il existe des correspondances entre formations et emplois qui optimisent les avantages pour les individus et les employeurs. Mais c'est la répartition des appariements qui fonde l'importance de la correspondance entre une formation et un emploi et non plus la correspondance normative entre une formation et un emploi qui dicte la viabilité des appariements observés.

Les jeunes sont (plus ou moins) aptes à occuper différents types d'emploi et, symétriquement, les emplois peuvent être occupés (plus ou moins bien) par des individus ayant suivi des formations différentes. Il est possible, au travers d'une enquête statistique, d'observer les appariements survenus entre les individus (issus d'une formation donnée) et les différents emplois qu'ils occupent.

L'ensemble de ces appariements peut être consigné dans une matrice formations-emplois. Il s'agit d'une banale table de contingence. Chaque ligne décrit la répartition des jeunes ayant suivi une même formation dans l'ensemble des emplois. C'est la loi de diffusion des formés. Chaque colonne décrit la diversité des recrutements pour une profession donnée. C'est la loi d'approvisionnement de la profession. Cette matrice constitue la seule réalité factuelle dont nous disposions sur la relation formations-emplois.

Dans cette représentation, la formation peut être définie par son niveau et sa spécialité, l'emploi par sa catégorie professionnelle et son domaine de spécialité. Il existe donc autant de lignes et de colonnes à la matrice qu'il y a de formations et d'emplois détaillés. La seule hypothèse réside dans les nomenclatures de collecte de l'information. La notion de diversité, consubstantielle à celle de compétence, est prise en compte aussi finement que le permet l'information disponible.

L'observation de ces matrices formations-emplois amène deux remarques fondamentales :

Elles n'ont rien d'aléatoire. Chaque formation conduit à un certain nombre d'emplois distincts. Réciproquement, les entreprises, en fonction des postes à pourvoir, recrutent des individus provenant de formations distinctes. Il y a donc bien une relation (statistique) entre formation reçue et emploi occupé. La matrice formations-emplois le démontre. Elle contient toute l'information recueillie sur cette relation.

Cependant, il n'est jamais possible - quelles que soient les manipulations de nomenclature en termes de niveau et/ou de spécialité - de mettre en évidence une relation biunivoque entre formations et emplois. La relation formations-emplois existe mais ce n'est pas une relation de causalité. Nous devons, pour nous mettre en conformité avec les enseignements récurrents des enquêtes, renoncer à dire qu'un recrutement se fait « parce que » l'individu a reçu telle formation. Nous devons nous contenter de dire que le recrutement qu'on observe s'est fait « sachant que » l'individu a reçu cette formation.

Ces deux remarques sont à l'origine de la démarche entreprise ici: placer la diversité au centre de l'analyse plutôt que la considérer comme une imperfection du marché; traiter empiriquement cette diversité.

\section{Les indices de Gini :} un outil simple pour caractériser I'appariement formations-emplois

Pour décrire les relations statistiques observées entre les « lignes formation » et les colonnes « emploi» de la matrice, nous avons choisi des indices de concentration de Gini ( $c f$. encadré 1). Ces indices ont déjà été utilisés par d'autres analystes des relations formationsemplois (Dumartin 1997; Torres, Chirache 2001; Heijke et alii 2003; Bruyère, Espinasse, Fourcade 2004 ; Chardon, 2005). Nous associons à chaque formation un indice de Gini (Gf) qui synthétise la loi de diffusion des jeunes ayant suivi cette formation dans l'ensemble des professions. Plus l'indice est élevé, plus les jeunes issus d'une formation ont trouvé 
un emploi dans un petit nombre de professions et plus les débouchés de cette formation sont concentrés sur ce petit nombre de professions. À chaque profession, nous associons un indice de Gini (Gp) synthétisant la loi d'approvisionnement des professions. Plus l'indice est élevé, plus le recrutement a été concentré sur quelques formations. Chaque appariement entre une formation et un type d'emploi se caractérise par un couple (Gf, Gp), dépendant des conditions du marché au moment de l'observation.

\section{Encadré 1 \\ Données, variables et modèles utilisés pour décrire la relation entre les formations et les emplois}

\section{Données}

Les données utilisées sont celles de l'enquête Génération 98 du Céreq. II s'agit d'un échantillon représentatif de l'ensemble des élèves et étudiants sortis du système de formation initiale en 1998 de tous niveaux (lycée et différents types d'enseignement supérieur) et de toutes spécialités académiques et/ou professionnelles. La base individus contient 55345 enregistrements. L'enquête interroge les jeunes sur leur parcours professionnel entre 1998 et 2001 . Chaque individu décrit tous les emplois qu'il a occupés pendant cette période, leur durée, les conditions de travail, la qualification, le niveau de rémunération à l'embauche (Céreq, 2002). La base emploi contient 101310 enregistrements. Ces enregistrements sont aussi des appariements entre un individu et chacun de ses emplois.

\section{Nomenclatures de formation et d'emploi}

La formation initiale d'un jeune est repérée à la fois par son niveau et sa spécialité. Avec les nomenclatures détaillées de l'enquête, on distingue 458 formations différentes, d'effectif non nul, croisant 8 niveaux de formation: Niveau 1 : diplômes $>=B A C+5$ ans; Niveau 2 : diplômes $B A C+3$, 4 ans; Niveau 3 : diplômes BAC +2 ; Niveau $4+: B A C+1,2$ ou 3 ans d'études supérieures non validées, Niveau 4 : BAC; Niveau 5 : année terminale de CAP/BEP ou sorties lycée; Niveau 5 bis: années non terminales de CAP ou BEP; Niveau 6 : Scolarité obligatoire avec la spécialité relativement détaillée (100 items environ).

Les emplois sont codés à l'aide de la nomenclature française des emplois de l'INSEE en 455 postes. Ils ont été regroupés en 162 familles professionnelles (sur la base des Familles professionnelles détaillées (Dares, 2006) pour obtenir, au niveau le plus détaillé possible, une homogénéité des taches constitutives de l'activité professionnelle et des effectifs raisonnables.

La matrice formations-emplois sur laquelle nous travaillons est donc de 458 lignes et 162 colonnes et contient 101310 appariements.

\section{Indices de Gini}

Pour une distribution de $N$ éléments $X_{i}$ classés par ordre croissant de ses arguments, et de moyenne $\mu$ l'indice de Gini que nous utilisons s'écrit :

$$
G=1-\frac{1}{2 \mu N^{2}} \sum_{i} \sum_{j}\left|X_{i}-X_{j}\right|
$$

L'indice de Gini d'une formation (Gf) est calculé à partir du fichier des emplois : tous les emplois pris par la cohorte à l'issue d'une formation donnée entrent dans le calcul de son indice de Gini, quel que soit le moment où l'emploi est pris et quelle que soit sa durée. L'indice de Gini d'une formation traduit la plus ou moins grande concentration de cette formation sur certaines familles professionnelles. C'est une caractéristique de la formation. Les indices des 458 formations repérées constituent une variable continue qui varie entre 0.684 et 1 . Plus l'indice est proche de 1 et plus les emplois pris par les jeunes de 
cette formation sont concentrés : il vaut 1 si tous les individus ont pris le même emploi ; il vaut 0.68 lorsque les emplois à l'issue de la formation connaissent la plus grande dispersion Cette variable est faiblement corrélée avec la durée des études.

L'indice de Gini d'un emploi (Gp) est calculé à partir de l'ensemble des formations (parmi les 458) qui ont conduit à cet emploi. Chaque emploi (famille professionnelle) possède son indice de Gini propre. La variable Gp varie continûment entre 0.788 et 0.999 . Les indices ne sont pas hiérarchisés par grandes catégories sociales: I'indice est maximum chez les Professions libérales (0.98) et très élevé chez les Ouvriers Qualifiés de type artisanal (0.97), nettement plus faible chez les ouvriers qualifiés de type industriel (0.93), par exemple.

\section{Variables d'adéquation normative}

Variables sur la spécialité : On a construit deux variables d'adéquation normative entre spécialité de formation et spécialité d'emploi à partir de deux tables de correspondance utilisées dans la littérature. La première (Adeq_gfe) repose sur les Groupes Formation-Emploi (Fourcade, Ourliac, Ourtau, 1992). Ces regroupements, construits sur la base d'un rapprochement des spécialités de formation professionnelle enseignées et puis mises en œuvre dans l'emploi et échangées sur le marché du travail, peuvent être appliqués aussi bien aux emplois qu'aux formations. Ils sont les mêmes pour tous les niveaux de formation. Un individu est déclaré adéquat (et la variable vaut 1) si sa spécialité de formation et sa spécialité d'emploi appartiennent au même GFE. La seconde table (Adeq_Bonnal) est issue d'un rapprochement terme à terme entre l'emploi détaillé et la spécialité de formation détaillée d'un individu. La correspondance est établie dès qu'il y a présomption d'une relation sectorielle ou fonctionnelle entre l'emploi et la spécialité. Cette table de passage a été réalisée par L. Bonnal (Bonnal et alii, 2005). Variables sur le niveau: Une variable d'adéquation normative entre le niveau de formation d'un individu et celui de son emploi a été construite sur la base du rapprochement normatif proposé par J. Affichard (Affichard, 1981 ). Elle a été croisée avec la variable d'adéquation de spécialité (Bonnal) afin d'obtenir un indicateur d'adéquation à la fois de niveau et de spécialité (Adeq_niveau*Bonnal).

Les jeunes issus d'une formation dont l'indice de Gini est élevé ont presque tous accédé au même petit nombre d'emplois. Ces jeunes ont pu choisir ces emplois parmi tous les emplois qui leur étaient accessibles, c'est-à-dire parmi tous les emplois pour lesquels leur formation délivre les compétences minimales requises. Ils ont obtenu ces emplois comme étant, pour eux, les meilleurs, c'est-à-dire ceux qui leur offraient le plus d'avantages, par exemple les mieux rémunérés. Quand au contraire Gf est faible, les jeunes de cette formation se sont dispersés sur un grand nombre d'emplois. Ceci montre que cette formation, tout en leur donnant accès à un grand nombre d'emplois, n'a pas permis à tous d'accéder aux meilleurs emplois (les plus avantageux, les mieux rémunérés), ou encore que tous les emplois présentaient des avantages équivalents à leurs yeux. Dans un contexte économique donné, l'indice de Gini positionne les formations entre elles. Plus une formation possède un indice de Gini élevé, plus il est probable qu'elle a permis aux étudiants d'accéder aux meilleurs emplois qui leur étaient accessibles. Inversement, plus une formation possède un indice de Gini faible et moins elle permet à l'ensemble des jeunes issus de cette formation d'accéder aux meilleurs emplois accessibles avec cette formation. L'indice de Gini d'une formation est une variable continue qui donne une mesure (ce n'est pas la seule) - aux conditions du marché - de la performance d'une formation dans l'accès à l'emploi.

Du côté des emplois, un Gp élevé signifie que ces emplois ont recruté des jeunes issus de quelques formations seulement. Ces formations ont fournimieux que les autres - à l'employeur les compétences qu'il cherchait ou qui lui ont permis de minimiser ses coûts de mise au travail. Ces formations ont doté les jeunes d'avantages concurrentiels sur ces emplois par rapport aux autres formations. $\mathrm{Au}$ contraire, un Gp faible indique qu' un grand nombre de formations délivre les compétences qui permettent l'accès à cet emploi. Ces formations sont concur- 
rentes pour cet emploi. L'indice Gp est donc un indicateur du degré de concurrence entre les formations pour fournir les compétences nécessaires à cet emploi. C'est aussi une mesure de la contrainte d'approvisionnement qu'ont supporté les entreprises pour cet emploi, toujours aux conditions d'offre et de demande à l'instant d'observation.

\section{DEUX APPROCHES POUR DEUX DESCRIPTIONS DES OBSERVATIONS}

Les données utilisées sont celles de l'enquête Génération 98 du Céreq, c'est-à-dire une enquête auprès des sortants de formation initiale (tous niveaux toutes spécialités) interrogés sur leur parcours professionnel (notamment leurs emplois successifs) pendant leurs trois premières années de vie active.

Les indices de Gini ont été calculés pour chaque formation et chaque emploi repérés au niveau le plus fin des nomenclatures disponibles ( $c f$. encadré 1).

Chaque embauche (chaque appariement ou encore chaque case de la matrice formations-emplois) peut être caractérisée par un couple (Gf, Gp) qui renseigne sur le degré de concentration de la formation $f$ d'un individu et sur celui de son emploi p. Les individus tels que Gf et Gp sont tous deux élevés ont reçu une formation qui leur permet d'accéder aux meilleurs emplois (parmi les emplois accessibles) et ont été embauchés dans un emploi nécessitant des compétences délivrées par quelques formations seulement. Il s'ensuit logiquement que, pour la majorité de ces individus, la correspondance formelle entre spécialité de formation et spécialité d'emploi doit être forte.

Ceci peut se vérifier empiriquement en listant les couples formations-emplois (i.e. les appariements) selon la valeur de leurs indices (Gf, Gp) (cf. tableau 1). Chaque case de ce tableau contient des exemples de couples (i. e. d'appariement) formations-emplois classés en fonction de la valeur de leurs indices de Gini². Les cas où les deux indices de

\footnotetext{
${ }^{2}$ Les indices de Gini sont des variables continues. Pour pouvoir lister les valeurs, on les a regroupés en tranches (basées sur les quantiles).
}

Gini sont maximum (i.e. proches de 1) correspondent, par exemple, aux couples (diplôme de niveau 1 Santé ; médecins) ou encore (diplôme de niveau 3 Santé ; Professions intermédiaires de la Santé (infirmiers, sage femmes, professions paramédicales)) c'est-à-dire des professions réglementées, ou encore (diplôme de niveau 3 Santé ; aide-soignante) ce qui correspond à un déclassement en termes de niveau dans l'approche normative. On y trouve également des couples (diplome de niveau 4 Coiffure; coiffeurs), montrant que, dans l'enquête Génération 98 au moins, la relation entre ce diplôme/spécialité et cette profession est très forte. Ces cas correspondent effectivement à des cas "d'adéquation normative » entre spécialité de formation et spécialité d'emploi.

À l'opposé, les couples (Gf, Gp) tels que les deux sont faibles désignent par exemple les recrutements d'Ouvrier Non Qualifié du tri ou de la manutention, des Militaires, des Agents de sécurité diplômés (de niveau V) ou non (Vbis) en mécanique-électricité, des Employés administratifs divers et des Employés de catégorie $\mathrm{C}$ de la Fonction publique diplômés du bac (N4) voire plus (N2) en Droit, Lettres Sciences Humaines ou encore de niveau 3 (N3) en Commerce. C'est-à-dire des appariements qui valorisent des compétences plus générales que professionnelles, ou qui révèlent une certaine indifférence (apparente) entre formation et emploi. L'adéquation entre spécialité de formation et d'emploi est ici nulle. Entre ces deux extrêmes, l'analyse des exemples de couples présents dans le tableau montre des relations formationsemplois plus ou moins fortes, soit que la formation diffuse au-delà de sa cible (par exemple Santé N4 ; secrétaire ou surveillant), soit que l'emploi recrute audelà de la formation « adéquate » (diplôme de niveau 1 en Chimie ; ingénieur informaticien).

Ces exemples sont là pour montrer que la description d'un appariement formation-emploi par son couple (Gf, Gp) paraît opérationnelle.

D'une manière plus générale, quel lien peut-on établir entre les valeurs associées aux couples (Gf, $\mathrm{Gp)}$ et le résultat binaire de l'approche normative (c'est-à-dire adéquat ou pas) ? Une formation dont l'indice de Gini est élevé débouche-t-elle plus souvent sur des situations d'adéquation? Un emploi dont l'indice de Gini est élevé fait-il appel aux formations dont les spécialités sont adéquates? 
Tableau 1

Exemples de couples (Formation ; Emploi) classés selon la valeur du couple (Gf, Gp)

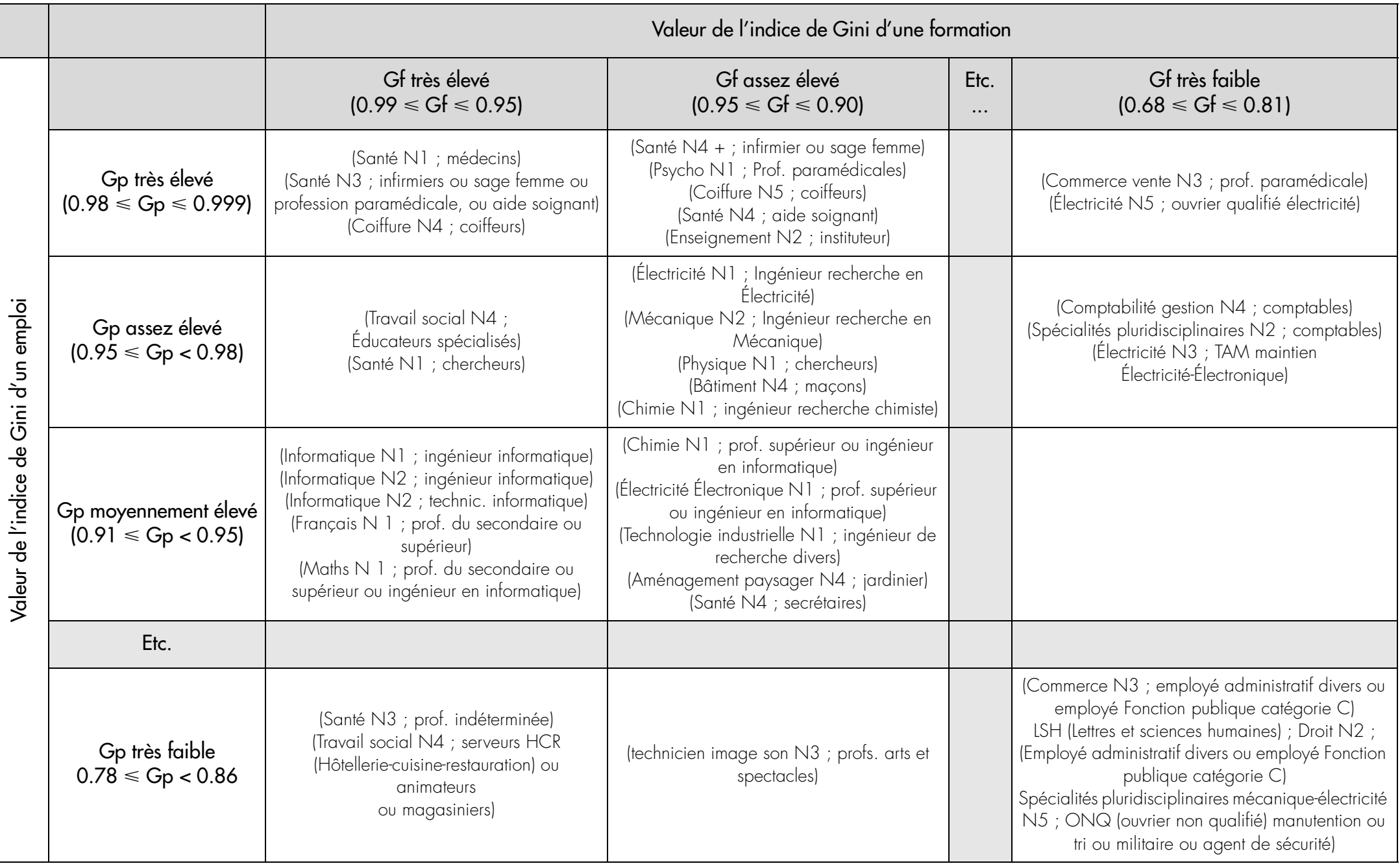

Note de lecture : au croisement d'une formation dont l'indice de Gini est très faible et d'un emploi dont l'indice de Gini est au contraire très élevé, on trouve, par exemple, les couples formationemploi suivants : des jeunes diplômés au niveau III en commerce (BTS ou DUT - brevet de technicien supérieur et diplôme universitaire de technologie) qui exercent des professions paramédicales, ou encore des diplomés de niveau 5 (CAP ou BEP - certificat d aptitude professionnelle et brevet détudes professionnelles) en électricité qui sont ouvriers qualifiés en électricité ; Ces deux formations ont des débouchés « dispersés » mais les emplois atteints dans ces deux exemples ont des recrutements très ciblés. $C f$. encadré 1 pour les significations de niveau (N1 à N6). 
Les variables normatives pour la correspondance entre spécialité de formation et spécialité d'emploi ont été calculées à l'aide de deux tables de correspondance différentes. L'une est basée sur les Groupes Formation-Emploi (Fourcade, Ourliac, Ourtau 1992), l'autre sur une correspondance fonctionnelle ou sectorielle établie terme à terme par Bonnal et al. (2005) ( $c f$. encadré 1).

Le taux «d'adéquats », au sens normatif, croit avec la valeur des indices de Gini (graphique 1). Plus l'un des deux indices est élevé, plus le taux est fort. Le taux est maximal (proche de $100 \%$ ) lorsque les deux indices sont proches du maximum (i.e. proches de 1). Un individu issu d'une formation très concentrée et qui est recruté sur un emploi recrutant de manière très ciblée a toutes les chances d'être «adéquat» au sens normatif. Ainsi, les professions réglementées sont à l'intersection des deux approches, métier et compétences : leurs lois de diffusion et d'approvisionnement sont qualitativement et quantitativement adaptées.

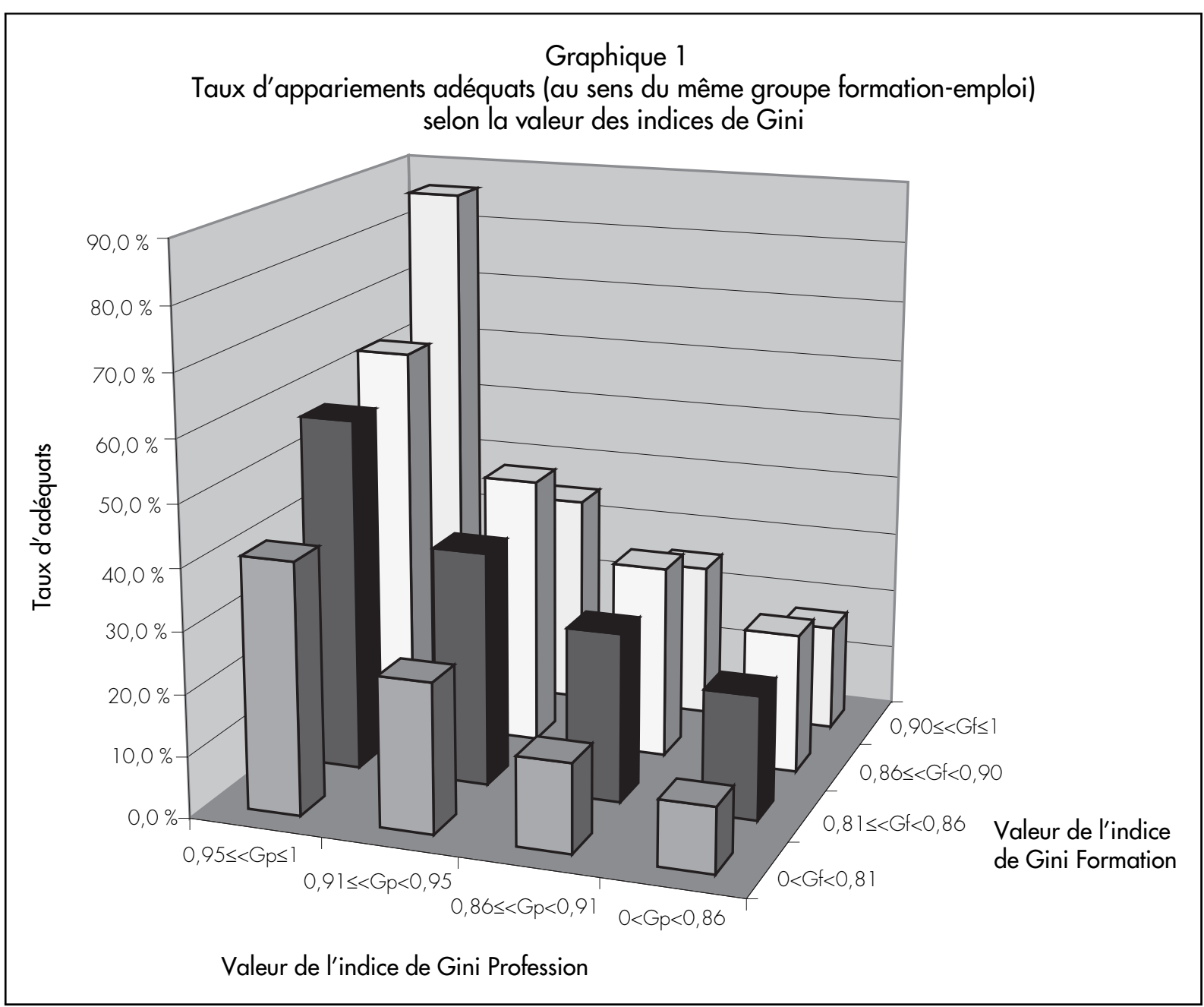

Note de lecture : les couples formation-emploi tels que Gf (indice de Gini formation) est très élevé (compris entre 0.95 et 1 ) et Gp (indice de Gini profession) très élevé (compris entre 0.90 et 1 ) sont adéquats au sens GFE à $86 \%$; ceux, au contraire, dont les deux indices Gf et Gp sont faibles (inférieurs à 0.81 et 0.86 ) ne sont que $10 \%$ à être adéquats.

Les deux approches aboutissent donc à des résultats disjoints mais surtout difficilement comparables : l'une fournit un couple de mesures continues du lien formation-emploi tandis que l'autre partage les situations d'emploi en deux : les adéquates et les non-adéquates. Les appariements déclarés «non adéquats» par l'approche normative peuvent être caractérisés par un couple d'indices de Gini relativement élevés. C'est le 
cas des diplômés de niveau 1 en chimie (DESS par exemple) qui sont ingénieurs en informatique. Ceci s'explique par l'extrême hétérogénéité de ces cas de non-adéquation: certains sont effectivement le mariage de la carpe et du lapin (c'est le cas du médecin acrobate ou, plus sérieusement, de la titulaire d'un diplôme de secrétariat, vendeuse de chaussures...); d'autres, beaucoup plus nombreux, peuvent se fonder sur l'échange de compétences non prévues lors de l'établissement de la table de passage entre formations et emplois adéquats (c'est l'exemple précité de l'ingénieur informaticien diplômé en chimie, mais aussi des diplômes commerciaux de niveau III menant à des professions paramédicales ou de BEP de mécaniciens devenus vendeurs de voitures). Par ailleurs, les appariements pour lesquels à la fois les deux indices de Gini sont élevés et l'adéquation normative réalisée font «masse » dans les statistiques descriptives (ils existent souvent). Les appariements hétéroclites sont au contraire uniques. Ils représentent peu d'individus et sont donc peu visibles parce que trop dispersés. Ainsi, les exemples fournis dans le tableau 1 ne concernent que les appariements représentés par au moins trente individus; si on lève cette barrière, les cas individuels peuvent être très hétéroclites.

\section{LES DEUX APPROCHES} À L'ÉPREUVE DE L'ANALYSE DES SALAIRES...

Nous avons vu que l'approche normative reposait sur la notion de métier et que la notion d'efficacité associée à cette approche était l'adéquation entre le niveau et la spécialité de formation et le niveau et la spécialité d'emploi. On s'attendrait à ce qu'un individu dont la formation est en adéquation avec l'emploi ait un gain supérieur à tous ceux qui n'ont $\mathrm{pu}$ trouver un tel emploi ${ }^{3}$. Dans cette première analyse, on ne tiendra pas compte des autres arbitrages possibles par rapport au salaire (vitesse d'accès, proximité géographique). Il suffit de caractériser par une variable la «bonne » adéquation de chaque appariement et de tester cette variable dans une équation de salaire. Deux tables de passage sont

\footnotetext{
3 On s'attend également à ce qu'il accède plus rapidement à l'emploi et dans de meilleures conditions (emploi stable par exemple). Ce modèle a été testé dans Béduwé et $a l$., 2005.
}

proposées pour caractériser l'adéquation en termes de spécialité, et nous avons utilisé celle proposée par Affichard (1981) pour mesurer l'adéquation en termes de niveau d'études ( $c f$. encadré 1).

L'approche statistique nous a conduit à calculer deux indices distincts Gf et Gp. Ces indices sont directement calculés à partir des observations et reflètent donc exclusivement le fonctionnement du marché (ses conditions de raretés relatives) au moment de l'enquête. Nous savons que l'indice Gf associé à une formation peut être vu comme un indicateur de l'avantage spécifique qu'une formation procure au jeune pour accéder à certains emplois. C'est donc un indicateur de la performance d'une formation. On s'attend à ce que les Gf soient positivement corrélés avec le salaire des jeunes issus de cette formation. Symétriquement, nous avons vu que Gp peut être vu comme une mesure de l'avantage que peuvent avoir les entreprises à cibler leur recrutement sur des formations spécifiques. On s'attend donc à ce que les Gp soient positivement corrélés avec le salaire des jeunes ayant obtenu cet emploi.

Nous avons testé les prévisions des deux approches dans une équation de salaire à l'aide des données de l'enquête Génération $98^{4}$.

Pour cela, nous avons construit un premier modèle d'explication des salaires ( $c f$. tableau $\mathbf{2}$, modèle $\mathbf{A})$ à partir des caractéristiques de l'individu (sexe, lieu d'étude, nombre d'années d'études, spécialité de formation, durée d'accès au premier emploi) et de données relatives à l'emploi occupé (horaires de travail, type de contrat de travail, date d'embauche), Dans cette configuration de base, nous avons introduit les indices de Gini (modèle B) puis les variables d'adéquation normative (modèle $\mathbf{C}$ ) et enfin les deux types de variables simultanément (modèle D) (tableau 2).

\footnotetext{
${ }^{4}$ On a utilisé une fonction de gain appliquée au logarithme du salaire d'embauche déclaré. On a choisi de travailler sur le fichier des séquences d'emplois, c'est-à-dire sur l'ensemble des recrutements observés lors de l'enquête Génération 98, pendant la période 1998-2001 $(\mathrm{N}=101310)$. Ce choix de considérer la totalité des emplois plutôt que le premier ou le dernier emploi ou encore l'emploi à la date de l'enquête, comme cela est couramment fait avec les enquêtes longitudinales, crée un biais dans l'échantillon (du fait de la répétition des individus ayant eu plusieurs emplois). Nous avons maintenu ce choix pour profiter de l'ensemble des appariements survenus et après avoir vérifié que tous les résultats restaient valables lorsque l'on procède plus classiquement.
} 
Le modèle de base $^{5}$ (tableau 2 , modèle A) fait apparaître des résultats classiques de l'analyse des rémunérations de jeunes débutants. Le salaire croit avec le nombre d'années d'études ; il est plus élevé pour les hommes et lorsque les études ont eu lieu à Paris. L'ancienneté sur le marché du travail (date d'embauche) procure un léger gain salarial alors qu'au contraire un accès retardé au premier emploi le diminue d'autant. Obtenir un emploi sur CDI augmente également le salaire d'embauche, tandis qu'un stage ou un emploi aidé le diminue fortement, par rapport à un contrat de type CDD. Enfin, le salaire est plus élevé dans les entreprises privées et de grande taille.

Les deux indices de Gini, formation et emploi, ont été introduits alternativement dans un premier temps (tableau 2, modèle B). Ils jouent dans le sens attendu: plus ils sont élevés et plus le salaire d'embauche est élevé. Ainsi, sortir d'une formation dont les débouchés sont concentrés sur peu d'emplois permet effectivement d'accéder aux emplois les mieux payés. Réciproquement, être recruté sur un emploi qui ne recrute qu'auprès d'un nombre restreint de formations permet d'obtenir un salaire en moyenne supérieur ${ }^{6}$.

Ces résultats sont d'autant plus remarquables que le modèle tient compte du niveau de formation (nombre d'années d'études) bien sûr, mais aussi de la spécialité de formation détaillée et de la taille d'entreprise. L'influence de ces indices est donc relativement forte.

L'introduction simultanée des deux indices de Gini confirme les rôles respectifs de chacun des indices ainsi que leur complémentarité7. Sortir d'une formation qui permet d'accéder aux meilleurs emplois parmi les emplois possibles et être embauché sur un

\footnotetext{
5 Plusieurs configurations de la variable décrivant le niveau d'étude de l'individu ont été testées; elles donnent des résultats très comparables (Béduwé, Espinasse, Vincens, 2005).

${ }^{6}$ L'indice de formation permet d'expliquer $19 \%$ d'écart de salaire entre les jeunes dont la formation possède l'indice de Gini le plus faible $(0.68)$ et ceux au contraire qui sortent d'une formation où l'indice est maximum (1), toutes les autres caractéristiques étant égales par ailleurs. L'indice de Gini profession explique $17 \%$ d'écarts de salaire entre les jeunes dont l'emploi (qui n'est pas dans le modèle) a l'indice de Gini le plus bas $(0.79)$ et celui dont l'indice de Gini est le plus élevé (1), toutes les autres caractéristiques étant là aussi égales par ailleurs.

${ }^{7}$ Les deux indices sont relativement peu corrélés $r=0.33$ et $\mathrm{r} 2=0.10$
}

emploi qui ne s'alimente qu'auprès de quelques formations concurrentes constitue bien un double bonus en termes de rémunération ${ }^{8}$.

Les modèles suivants introduisent les variables d'adéquation normatives $(C)$. L'adéquation en termes de spécialité de formation et d'emploi apporte $3 \%$ de bonus de salaire moyen avec la norme GFE, $6 \%$ avec la table proposée par L. Bonnal et $15 \%$ lorsque l'adéquation se fait sur le niveau et la spécialité ( $c f$. encadré $\mathbf{1}$ et tableau 2, colonne $\mathbf{C}$ ). Ces résultats confirment ceux obtenus par Bruyère et Lemistre (2005) sur le «faible» gain lié à l'adéquation de spécialité et sur le fait qu'il varie fortement en fonction du niveau de formation. Les deux variables d'adéquation normative (GFE et Bonnal) donnent des résultats qui varient du simple au double. Cela illustre la difficulté d'établir, «à dire d'experts », la correspondance normative entre formations et emplois.

Le modèle D combine les approches statistique et normative. Ces approches étant conceptuellement différentes, la confrontation directe des coefficients doit être menée avec prudence. Les variables normatives sont dichotomiques. Les appariements « adéquats » ont une prime salariale. Tous les autres appariements sont considérés comme identiquement pénalisants. Les variables statistiques sont continues. Elles rendent compte des observations et tiennent compte du fait qu'occuper un emploi « inadéquat» n'est pas (toujours) pénalisant en termes de salaire.

On constate que les deux types de variable (Gini et normative) introduits ensemble sont significatifs.

Les coefficients de Gini n'épuisent pas l'information fournie par la variable d'adéquation normative et réciproquement. L'adéquation n'est qu'un cas particulier des appariements présentant, le plus souvent, un couple (Gf, Gp) élevé. Ce cas particulier n'est pas toujours repéré en tant que tel par les Gini (ce n'est pas leur objectif...). On peut considérer que ces deux variables se complètent. En revanche, si la norme Bonnal voit un avantage supplémentaire à l'adéquation a priori des spécialités $(+4 \%$, modèle $\mathrm{D})$, la norme GFE, au contraire, est maintenant faiblement

\footnotetext{
${ }^{8}$ Ce bonus peut aller jusqu'à $32 \%$ pour un tel individu par rapport à un individu qui, toutes choses égales par ailleurs, sortirait au contraire d'une formation au Gini faible et recruté sur un emploi dont l'indice est également faible.
} 
Tableau 2

Variables expliquant le salaire d'embauche des jeunes de génération 98 entre 1998 et 2001

\begin{tabular}{|c|c|c|c|c|c|c|c|c|c|c|}
\hline \multirow{3}{*}{$\begin{array}{l}\begin{array}{l}\text { Variable expliquée : Logarithme du salaire } \\
\text { mensuel primes incluses(1) }\end{array} \\
\text { Homme }\end{array}$} & \multirow{3}{*}{$\begin{array}{c}\begin{array}{c}\text { Modèle de } \\
\text { Base (A) }\end{array} \\
9 \%(2) \\
\end{array}$} & \multicolumn{3}{|c|}{$\begin{array}{c}\text { Modèles B } \\
\text { Indices de Gini }\end{array}$} & \multicolumn{3}{|c|}{$\begin{array}{c}\text { Modèles } C \\
\text { Variables d'adéquation } \\
\text { normative }\end{array}$} & \multicolumn{3}{|c|}{$\begin{array}{l}\text { Modèles D } \\
\text { Mixte }\end{array}$} \\
\hline & & \multicolumn{8}{|c|}{ Rendement salarial en \% } & \\
\hline & & $9 \%$ & $9 \%$ & $8 \%$ & $9 \%$ & $8 \%$ & $8 \%$ & $8 \%$ & $8 \%$ & $8 \%$ \\
\hline Nombre d'années d'études & $6 \%$ & $5 \%$ & $6 \%$ & $5 \%$ & $6 \%$ & $5 \%$ & $5 \%$ & $5 \%$ & $5 \%$ & $5 \%$ \\
\hline \multicolumn{11}{|l|}{ Spécialité de formation } \\
\hline Sciences & $4 \%$ & $4 \%$ & $3 \%$ & $3 \%$ & $5 \%$ & $5 \%$ & $3 \%$ & $3 \%$ & $4 \%$ & $2 \%$ \\
\hline Sciences Humaines et droit & $-6 \%$ & $-4 \%$ & $-5 \%$ & $-4 \%$ & $-5 \%$ & $-5 \%$ & $-4 \%$ & $-4 \%$ & $-3 \%$ & $-3 \%$ \\
\hline Lettres & $-6 \%$ & $-5 \%$ & $-6 \%$ & $-5 \%$ & $-5 \%$ & $-5 \%$ & $-4 \%$ & $-5 \%$ & $-4 \%$ & $-4 \%$ \\
\hline Technologie & $4 \%$ & $7 \%$ & $3 \%$ & $5 \%$ & $5 \%$ & $4 \%$ & $3 \%$ & $5 \%$ & $5 \%$ & $4 \%$ \\
\hline Industriel divers & $1 \%$ & $-2 \%$ & $0 \%$ & $-3 \%$ & $1 \%$ & $1 \%$ & ns & $0 \%$ & $-2 \%$ & $-2 \%$ \\
\hline Mécanique - électronique & $1 \%$ & $3 \%$ & ns & $1 \%$ & $2 \%$ & $1 \%$ & $1 \%$ & $1 \%$ & $1 \%$ & $1 \%$ \\
\hline Santé & $17 \%$ & $11 \%$ & $11 \%$ & $5 \%$ & $16 \%$ & $16 \%$ & $12 \%$ & $5 \%$ & $6 \%$ & $4 \%$ \\
\hline Autres services aux personnes & $1 \%$ & $-2 \%$ & $0 \%$ & $-4 \%$ & $1 \%$ & $1 \%$ & ns & $-4 \%$ & $-4 \%$ & $-3 \%$ \\
\hline Services aux collectivités & $-3 \%$ & $-5 \%$ & $-3 \%$ & $-4 \%$ & $-2 \%$ & $-2 \%$ & $-1 \%$ & $-5 \%$ & $-4 \%$ & $-3 \%$ \\
\hline Économie et gestion & Ref, & Ref, & Ref, & Ref, & Ref, & Ref, & Ref, & Ref, & Ref, & Ref, \\
\hline Lieu de formation: Paris & $7 \%$ & $7 \%$ & $7 \%$ & $7 \%$ & $7 \%$ & $7 \%$ & $7 \%$ & $7 \%$ & $7 \%$ & $7 \%$ \\
\hline Temps d'accès au ler emploi & $0 \%(3)$ & $0 \%$ & $0 \%$ & $0 \%$ & $0 \%$ & $0 \%$ & $0 \%$ & $0 \%$ & $0 \%$ & $0 \%$ \\
\hline Date de prise d'emploi (en mois) & $0 \%$ & $0 \%$ & $0 \%$ & $0 \%$ & $0 \%$ & $0 \%$ & $0 \%$ & $0 \%$ & $0 \%$ & $0 \%$ \\
\hline \multicolumn{11}{|l|}{ Horaires de travail } \\
\hline Temps plein & Ref, & Ref, & Ref, & Ref, & Ref, & Ref, & Ref, & Ref, & Ref, & Ref, \\
\hline Moins d'un mi-temps & $-45 \%(4)$ & $-45 \%$ & $-45 \%$ & $-45 \%$ & $-45 \%$ & $-45 \%$ & $-45 \%$ & $-45 \%$ & $-45 \%$ & $-45 \%$ \\
\hline Mi-temps & $-34 \%$ & $-34 \%$ & $-34 \%$ & $-34 \%$ & $-34 \%$ & $-34 \%$ & $-34 \%$ & $-34 \%$ & $-34 \%$ & $-34 \%$ \\
\hline 3 jours par semaine environ & $-33 \%$ & $-33 \%$ & $-32 \%$ & $-32 \%$ & $-33 \%$ & $-32 \%$ & $-32 \%$ & $-32 \%$ & $-32 \%$ & $-32 \%$ \\
\hline 4 jours par semaine environ & $-22 \%$ & $-22 \%$ & $-21 \%$ & $-21 \%$ & $-22 \%$ & $-21 \%$ & $-21 \%$ & $-21 \%$ & $-21 \%$ & $-21 \%$ \\
\hline
\end{tabular}


Tableau 2 (suite)

\begin{tabular}{|c|c|c|c|c|c|c|c|c|c|c|}
\hline \multicolumn{11}{|l|}{ Type de contrat de travail } \\
\hline Contrat à Durée Indéterminée & $8 \%$ & $8 \%$ & $7 \%$ & $8 \%$ & $7 \%$ & $7 \%$ & $7 \%$ & $7 \%$ & $6 \%$ & $8 \%$ \\
\hline Contrat à Durée Déterminée & Ref, & Ref, & Ref, & Ref, & Ref, & Ref, & Ref, & Ref, & Ref, & Ref, \\
\hline Stage & $-5 \%$ & $-5 \%$ & $-4 \%$ & $-5 \%$ & $-5 \%$ & $-5 \%$ & $-5 \%$ & $-4 \%$ & $-4 \%$ & $-5 \%$ \\
\hline Mesure pour l'emploi & $-20 \%$ & $-20 \%$ & $-20 \%$ & $-21 \%$ & $-20 \%$ & $-20 \%$ & $-21 \%$ & $-21 \%$ & $-20 \%$ & $-21 \%$ \\
\hline \multicolumn{11}{|l|}{ Secteur d'activité } \\
\hline Secteur Public & $3 \%$ & $3 \%$ & $4 \%$ & $3 \%$ & $3 \%$ & $3 \%$ & $2 \%$ & $4 \%$ & $3 \%$ & $3 \%$ \\
\hline Grande Entreprise & $14 \%$ & $14 \%$ & $14 \%$ & $14 \%$ & $14 \%$ & $14 \%$ & $13 \%$ & $14 \%$ & $14 \%$ & $13 \%$ \\
\hline Petite ou Moyenne Entreprise & Ref, & Ref, & Ref, & Ref, & Ref, & Ref, & Ref, & Ref, & Ref, & Ref, \\
\hline Très petite entreprise & $-7 \%$ & $-7 \%$ & $-8 \%$ & $-8 \%$ & $-7 \%$ & $-7 \%$ & $-7 \%$ & $-8 \%$ & $-8 \%$ & $-8 \%$ \\
\hline Non réponse & Ns & Ns & Ns & Ns & Ns & Ns & Ns & Ns & Ns & Ns \\
\hline Gini_formation & & $19 \%(5)$ & & $16 \%$ & & & & $16 \%$ & $16 \%$ & $13 \%$ \\
\hline Gini_profession & & & $17 \%(6)$ & $16 \%$ & & & & $17 \%$ & $13 \%$ & $9 \%$ \\
\hline Adeq_gfe & & & & & $3 \%$ & & & $0 \%$ & & \\
\hline Adeq_bonnal & & & & & & $6 \%$ & & & $4 \%$ & \\
\hline \multicolumn{11}{|l|}{ Adeq_niveau *bonnal } \\
\hline Niveau et spécialité & & & & & & & $15 \%$ & & & $12 \%$ \\
\hline Niveau seul & & & & & & & $8 \%$ & & & $7 \%$ \\
\hline Spécialité seule & & & & & & & $3 \%$ & & & $1 \%$ \\
\hline Pas d'adéquation & & & & & & & Ref, & & & Ref, \\
\hline \% de variance des salaires expliquée R2 & $52 \%(7)$ & $52 \%$ & $52 \%$ & $53 \%$ & $52 \%$ & $52 \%$ & $54 \%$ & $53 \%$ & $53 \%$ & $54 \%$ \\
\hline Effectif & 98790 & 98790 & 9890 & 98790 & 98790 & 98790 & 98790 & 98790 & 98790 & 98790 \\
\hline
\end{tabular}

(1) La fonction de gain est une régression linéaire du logarithme du salaire sur un ensemble de variables. Elle permet de quantifier l'apport salarial des différentes modalités de chaque variable.

(2) Lire : les hommes gagnent en moyenne $9 \%$ de plus que les femmes.

(3) Bien que significatifs à $1 \%$, les coefficients sont très proches de 0 , d'où l'arrondi à $0 \%$.

(4) Lire : les individus qui travaillent à moins d'un mi-temps ont en moyenne un salaire inférieur de $45 \%$ par rapport aux individus à temps plein (Réf.).

(5) Lire : les individus qui sortent d'une formation dont l'indice de Gini est maximum (1) gagnent en moyenne $19 \%$ de plus que ceux qui sortent d'une formation dont l'indice de Gini est minimum (0.68).

(6) Lire : obtenir un emploi dont l'indice de Gini est maximum (1) procure un gain salarial moyen de $17 \%$ par rapport à un emploi dont l'indice de Gini est minimum (0.79).

(7) Signifie que $52 \%$ de la variance des salaires observés dans l'enquête s'explique par l'ensemble des variables retenues dans le modèle. Ce taux est normal compte tenu des spécifications du modèle. Source : Enquête Génération 98 (CEREQ), base emplois, hors temps de travail non déclarés, calculs des auteurs. 
significative et même négativement corrélée au salaire $(0 \%$, modèle $\mathrm{D})$. La formalisation des relations formations-emplois à partir de normes $a$ priori aboutit à une évaluation dépendante de la norme retenue.

On pourrait répondre à cela que les indices de Gini dépendent aussi des nomenclatures de spécialité, de formation comme d'emploi, utilisées. Les résultats présentés ici utilisent ces nomenclatures au niveau le plus fin disponible dans l'enquête. La robustesse de ces résultats a été testée dans plusieurs directions (Béduwé et al. 2005). Quelle que soit la précision avec laquelle on définit la formation pour le calcul de Gini (et donc la précision du calcul de Gini) et quelle que soit la spécification retenue pour caractériser la formation d'un individu dans les modèles (niveau d'étude ou nombre d'années d'études ou classe/ diplôme de sortie), les coefficients des indices de Gini sont toujours significatifs, positifs, et l'amplitude des écarts de salaire expliqués relativement constante.

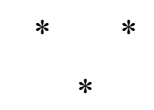

En partant du principe qu'une formation est caractérisée à la fois par son «niveau » (sa durée, son type) et par sa spécialité et qu'un emploi l'est également par sa catégorie sociale et son domaine de spécialité, l'analyse des relations entre formations et emplois oblige à considérer l'appariement entre un individu et son emploi dans ces deux dimensions. Les travaux qui prennent en compte cet impératif relèvent de deux conceptions différentes de la relation entre formations et emplois : l'une, normative, est construite autour de la notion de métier; l'autre, statistique, s'articule autour de la notion de compétence. Elles aboutissent à des formalisations que nous avons testées et confrontées. Dans les deux cas, une bonne correspondance entre formation reçue et emploi exercé est efficace en termes salariaux. L'ampleur du rendement estimé est cependant différente. Ces différences de résultat s'expliquent.

L'approche normative postule et prouve qu'une adéquation normative des compétences est efficace. Mais elle traite tous les autres cas comme identiquement non optimaux. Techniquement, ceci conduit à rejeter du calcul, au motif qu'ils ne sont pas adéquats, un grand nombre d'appariements performants. D'où le faible différentiel de gain en faveur des situations d'adéquation.

L'approche statistique paraît mieux adaptée pour analyser l'ensemble des relations entre formations et emplois. Elle part de l'idée que ces relations s'établissent au moment où les contrats de travail se concluent et non pas a priori. Elle s'appuie sur l'observation de la diversité des matrices formationsemplois, diversité engendrée par la substituabilité partielle des compétences et donc des travailleurs. Cette diversité n'est pas exempte de régularités qui témoignent de l'existence de correspondances entre formations et emplois et que nous avons analysées à l'aide d'indices de concentration (indices de Gini). Cette approche montre qu'une formation débouchant sur un nombre de cibles professionnelles restreintes offre des rendements de l'éducation élevés (salaires). Plus la cible est restreinte, plus les rendements sont élevés.

Les deux approches peuvent apparaître complémentaires. Sortir d'une formation performante au sens de Gini procure un avantage général qui peut se cumuler avec un avantage spécifique lorsque l'on est amené à exercer le métier adéquat tel que décrit par la table de passage. Certaines formations confèrent effectivement un avantage absolu (monopolistique) pour accéder à un emploi donné. C'est ce que signale la variable normative. Mais d'autres formations peuvent procurer des compétences substituables, moyennant un coût d'adaptation un peu plus élevé. C'est ce que signale l'indice de Gini.

L'approche normative a priori apparaît donc comme un cas particulier d'allocation des compétences. Elle ne peut en revanche prétendre à modéliser l'ensemble des relations entre formations et emplois. Elle repose fondamentalement sur la notion de métier qui n'a plus, dans nos économies modernes, l'importance qu'elle pouvait avoir dans les économies préindustrielles. La notion de métier - parce qu'elle cherche à distinguer formellement les emplois entre eux - ne peut tenir compte d'emplois aux contours rendus plus flous du fait d'une division du travail complexe. De ce point de vue, la notion de compétences paraît plus satisfaisante car basée sur un 
principe plus général et réaliste d'allocation des jeunes aux emplois.

Enfin, l'approche statistique proposée permet de relativiser la distinction entre formations générales et professionnelles. Elle suggère que les formations pourraient être utilement classées dans un continuum selon leur degré de professionnalité 9 . Cette professionnalité peut être caractérisée par le constat de débouchés professionnels plus ou moins

${ }^{9}$ Cette idée a été défendue par Giret et Moullet (2002) à partir d'une analyse différente reposant sur la définition de la professionnalisation d'une formation dans l'enseignement supérieur proposée par Vincens et Chirache (1996). concentrés. Nos résultats montrent que cette méthode est efficace puisque associée à des rendements positifs de la formation. Cette définition $a d$ hoc de la professionnalité d'une formation est distincte de la définition administrative a priori des formations professionnelles - même si on peut espérer qu'elles se recoupent largement. Elle est purement empirique et correspond à la réalité observée a posteriori. Elle est donc particulièrement utile dans une optique de mesure de la performance puisqu'elle permet de confronter les objectifs d'une politique (la professionnalisation) avec ses résultats.

\section{BIBLIOGRAPHIE}

Affichard J. (1981), «Quels emplois après l'école : la valeur des titres scolaires depuis $1973 »$, Économie et Statistique, 173, pp. 7-26.

Béduwé C., Espinasse J.-M. \& Vincens J. (2005), «Spécialité de formation, spécialité d'emploi et performance d'insertion : logique de métier v/s logique compétences », Note Lirhe n ${ }^{\circ} 413$ (05-08), 28 p.

Bruyère M. \& Lemistre P. (2005), Trouver un emploi en rapport avec sa formation: une situation rentable?, in Giret, Lopez \& Rose (Éds), Quelles formations pour quels emplois?, Paris, La Découverte, pp. 249-262.

Bruyère M., Espinasse J.-M. \& Fourcade B. (2004), Fluctuations du marché et insertion des jeunes : conséquences sur la politique des diplômes, Rapport de recherche pour le ministère de l'Éducation nationale, Direction de l'enseignement scolaire, $214 \mathrm{p}$.

Bonnal L., Boumahdi R., Favard P. \& Mendes S. (2005) «Quelle relation formation-emploi pour les jeunes de niveau $V$ ? Une comparaison entre les sortants d'apprentissage et de lycée professionnel », in
Giret, Lopez \& Rose (Éds), Quelles formations pour quels emplois ?, Paris, La Découverte, pp. 126-144.

Céreq (Éd.) (2002), Quand l'école est finie. Premiers pas dans la vie active de la génération 98, Marseille, Céreq, $75 \mathrm{p}$.

Chardon O. (2005), La correspondance formationemploi sous l'éclairage de la gestion des âges dans les métiers, in Giret, Lopez \& Rose (Éds), Quelles formations pour quels emplois?, Paris, La Découverte, pp. 163-178.

Couppié T., Giret J.-F., Lopez A. (2005), « Des formations aux premiers emplois : une correspondance plutôt mal assurée », in Giret, Lopez \& Rose (Éds), Quelles formations pour quels emplois ?, Paris, La Découverte, pp. 79-96.

Cour de Justice des Communautés européennes (1985), Arrêt Gravier. 293/83, Recueil 1985.

Dares, (2006), Les Familles Professionnelles, Nomenclature FAP-2003, table de correspondance FAP/PCS/ROME.

Dumartin S. (1997) «Formation emploi : quelle adéquation? », Économie et Statistique (303), pp. 59-80. 
Fourcade B., Ourliac G. \& Ourtau M. (1992), «Les groupes formation-emploi GFE : une nomenclature pour l'analyse de la relation formation-emploi dans les régions ", L'Orientation scolaire et professionnelle (4/1992), pp. 383-410.

Frétigné C. (2006), " Note critique», Revue française de pédagogie, $\mathrm{n}^{\circ} 155$, avril-mai-juin.

Giret J.-F. \& Moullet S. (2002), « Retour sur la professionnalisation de l'enseignement supérieur », Communication aux Journées d'étude du RAPPE, Aix-en-Provence, 21-22 novembre.

Giret J.-F., Moullet S. et Thomas G. (2003), «L'enseignement supérieur professionnalisé. Un atout pour entrer dans la vie active », Bref-Céreq $\mathrm{n}^{\circ} 195$, mars.

Giret J.-F. \& Moullet S. (2005), «L'adéquation formation emploi après les filières professionnelles de l'enseignement supérieur », in Giret, Lopez \& Rose (Éds), Quelles formations pour quels emplois?, Paris, La Découverte, pp. 111-125.

Giret J.-F., Lopez A., Rose J. (2005), Quelles formation pour quels emplois?, Ouvrage collectif, Paris, La Découverte, $384 \mathrm{p}$.
Heijke H., Matheeuwsen A. \& Willems E. (2003), "Clustering educational categories in a heterogeneous labour market", Education Economics, 11 (1), pp. 89-108.

Kirsch J.-L. \& Werquin, P. (1995), « Quelque part... une relation formation-emploi », Formation Emploi, $\mathrm{n}^{\circ} 52$, pp. 29-47.

Stankiewicz F. (2006), Note de lecture, Travail et Emploi $\mathrm{n}^{\circ}$ 107, juillet-septembre.

Torres D. \& Chirache S. (2001), « Analyse des liens formation-emploi chez les jeunes débutants », Note d'information du ministère de l'Éducation nationale 01(60), $6 \mathrm{p}$.

Vincens J. (2005), « L'adéquation formation-emploi », in Giret, Lopez \& Rose (Éds), Quelles formations pour quels emplois ?, Paris, La Découverte, pp. 149162.

Vincens J. \& Chirache S. (1996), « Professionnalisation des enseignements supérieurs", in Professionnaliser les formations : choix ou nécessité?, Haut Comité éducation-économie (HCEE), Paris, La Documentation française, collection «Rapports officiels », pp. 143-182. 


\section{Résumé}

\section{De la formation professionnelle à la professionnalité d'une formation}

Catherine Béduwé, Jean-Michel Espinasse, Jean Vincens

La professionnalisation des formations s'accompagne d'une diversification croissante des spécialités enseignées. Prendre en compte ces spécialités dans l'analyse des relations entre formations et emplois devient nécessaire à l'évaluation des politiques éducatives. Deux approches sont confrontées dans cet article. La première est fondée sur la mise en correspondance a priori des formations et des emplois. L'adéquation est considérée comme une condition de la performance. La seconde part de l'observation des recrutements survenus au cours de l'insertion professionnelle et propose d'analyser la diffusion de chaque formation entre les emplois et l'alimentation des emplois par les formations. Les indices de concentration (de Gini), qui caractérisent alors chaque formation et chaque emploi, sont introduits dans des équations de salaire. Le test des deux approches sur un même jeu de données illustre l'intérêt de la seconde.

\section{Mots-clés}

Compétence, contenu de formation, enquête Génération 98, évaluation, professionnalisation de l'enseignement, relation formation-emploi, salaire.

Journal of Economic Literature : J 31 , J 24 


\section{Cahiers du Genre-n 43/2007}

(à paraître en octobre 2007)

\section{- Dossier}

\section{Genre, féminisme et valeur de l'art \\ Coordonné par \\ Séverine Sofio, Perin Emel Yavuz et Pascale Molinier}

口 Séverine Sofio, Perin Emel Yavuz et Pascale Molinier

L'art au prisme du genre : la valeur en question (Introduction)

- Fabienne Dumont et Séverine Sofio

Esquisse d'une épistémologie de la théorisation féministe en art

a Griselda Pollock

Des canons et des guerres culturelles

a Patricia Mainardi et Mathilde Ferrer (Entretien)

De chaque côté de l'Atlantique, deux parcours féministes en art

(Propos recueillis par Fabienne Dumont, Séverine Sofio et Perin Emel Yavuz)

Myriem Naji

Valeur des tapis marocains : entre productrices d'artisanat et marchands d'art

a Maria Antonietta Trasforini

Du génie au talent : quel genre pour l'artiste?

- Françoise Frontisi-Ducroux

'La fille de Dibutade', ou l'inventrice inventée

$\square$ Frédérique Villemur

Pensée queer et mélancolie du genre

- Elvan Zabunyan

Histoire de l'art contemporain et théories féministes : le tournant de 1970

a David Zerbib

Le masculin et la performance de l'universel

\section{- Hors-champ}

Roland Pfefferkorn

Autour de l'organisation d'une journée d'étude sur la prostitution. L'impossible compromis

\section{- Notes de lecture}

Vente au numéro : à la librairie L'Harmattan et dans les librairies spécialisées Les abonnements sont annuels et partent du premier numéro de l'année en cours :

France : $45 €$ - Étranger : $50 €$ frais d'envoi compris Veuillez adresser votre commande

aux éditions L'Harmattan - 5-7 rue de l'École Polytechnique 75005 Paris

Tél. 0140467920 - Mél diffusion-harmattan@wanadoo.fr

Pour tout autre renseignement sur la revue, s'adresser au secrétariat de rédaction :

Danièle Senotier

tél. 0140251165 - mél. daniele.senotier@gtm.cnrs.fr 